\title{
La perspective européenne
}

\section{Michael Vorbeck}

\section{OpenEdition}

\section{Journals}

Édition électronique

URL : http://journals.openedition.org/ries/3293

DOI : 10.4000/ries.3293

ISSN : 2261-4265

\section{Éditeur}

Centre international d'études pédagogiques

\section{Édition imprimée}

Date de publication : 1 septembre 1996

Pagination : 127-134

ISSN : 1254-4590

\section{Référence électronique}

Michael Vorbeck, «La perspective européenne », Revue internationale d'éducation de Sèvres [En ligne],

11 | 1996, mis en ligne le 29 juillet 2013, consulté le 01 mai 2019. URL : http:// journals.openedition.org/ries/3293 ; DOI : 10.4000/ries.3293

Ce document a été généré automatiquement le 1 mai 2019.

(c) Tous droits réservés 


\title{
La perspective européenne
}

\author{
Michael Vorbeck
}

1 Depuis qu'il y a des écoles en Europe, il y a aussi eu des formes d'évaluation des élèves. $\mathrm{Au}$ courant des siècles, différents systèmes de contrôle de connaissances se sont développés dans les différents pays. L'évaluation servait à des fins très diverses : constat de réussite ou d'échec dans un programme donné, base de décision d'admission à un cycle supérieur, sélection pour l'accès à un certain type d'établissement scolaire ou supérieur ou une certaine filière, moyen pour l'élève de se situer lui-même par rapport aux autres ou par rapport au niveau exigé, etc.

2 À la suite des réformes scolaires des années 1960 et 1970, une tendance vers l'abolition des notes scolaires traditionnelles, au moins dans l'enseignement primaire, s'est manifestée en Grande-Bretagne, dans certains Länder allemands et dans les pays nordiques. L'idée était de remplacer les notes précises par un système d'évaluation plus globale et sommaire.

3 Avec l'intégration européenne et la mobilité croissante de familles et d'individus d'un pays à l'autre, le besoin a été ressenti d'arriver à une comparaison des différents systèmes d'évaluation en vigueur afin de mieux situer le niveau d'un élève ou étudiant étranger, demandant l'accès à une école ou université du pays-hôte.

4 En ce qui concerne l'accès à l'enseignement supérieur, l'équivalence (formelle et juridique) des diplômes de fin d'études secondaires fut établie par une convention du Conseil de l'Europe, signée déjà en 1953 et toujours valable, mais les problèmes de transfert pendant la scolarité secondaire ou pendant un cursus universitaire restaient posés, même après la signature de deux autres conventions sur l'équivalence des périodes d'études.

5 En plus, les différentes réformes scolaires des pays européens soulevèrent des doutes en ce qui concerne l'équivalence réelle (matérielle) du certificat de fin d'études secondaires (baccalauréat, Abitur, Matura, etc.).

6 Un autre problème était le sentiment ou le constat d'échec scolaire suite à de mauvaises notes qui souvent s'avérait comme un véritable drame psychologique pour les élèves et leurs familles. 


\section{La contribution de la recherche}

7 Ces problèmes étant communs à de nombreux pays européens, la recherche en matière d'éducation s'est occupée de cette situation et a essayé de comparer les différents systèmes d'évaluation et d'en examiner la validité et les conséquences. La base de données EUDISED (European Documentation and Information System for Education) du Conseil de l'Europe, accessible en ligne directe par l'Agence spatiale européenne et la Biblioteca di documentazione pedagogica de Florence et également sur Internet, contient de nombreux résumés de projets de recherche à ce sujet. Les mots-clés du Thesaurus européen de l'Éducation (géré conjointement par le Conseil de l'Europe et la Commission européenne) permettent d'interroger cette base de données.

8 Suivant la suggestion de plusieurs États-membres, le Conseil de l'Europe prit l'initiative, à deux reprises, de tirer le bilan de toutes ces recherches afin de fournir des indications aux responsables de la politique scolaire, donc aux ministères de l'Éducation, ainsi qu'aux formateurs des enseignants et aux enseignants eux-mêmes. Deux ateliers de recherche pédagogique furent organisés à cette fin, l'un à Liège ${ }^{1}$ l'autre à Jyväskylä ${ }^{2}$ en Finlande. Dans les deux cas, la méthode de travail fut la même : six communications commandées, une série de rapports de recherche nationaux ou individuels, travaux en groupe, résumé final par un rapporteur général le matin du dernier jour. A l'atelier de Jyväskylä participèrent pour la première fois dans ce type de discussion les pays de l'Europe centrale et orientale ${ }^{3}$.

\section{L'identification des problèmes}

9 Lors des deux ateliers, de nombreux problèmes furent identifiés. Dans la plupart des systèmes scolaires, l'évaluation des élèves reste très subjective et relativement peu fiable, surtout dans les lettres et sciences humaines (langue maternelle, dissertation, langues étrangères, histoire, etc.). Beaucoup d'enseignants n'ont jamais été formés à une évaluation objective et vraiment professionnelle

10 Les notes restent souvent assez superficielles, disent trop peu ce que l'élève est réellement capable de faire. L'évaluation des connaissances d'un élève en anglais par un « assez bien » ou par " 11 sur 20 », par exemple, ne permet pas de savoir si l'élève arrive à prononcer l'anglais correctement et à communiquer oralement dans des situations de la vie quotidienne (demander un repas, demander son chemin, mener une conversation sur sa famille, son pays, etc.).

11 L'évaluation à l'école ne couvre grosso modo que $30 \%$ des connaissances, attitudes, comportements et compétences dont l'élève aura besoin dans la vie d'adulte. Dans beaucoup de pays, l'accent est encore trop mis sur la répétition mécanique de connaissances encyclopédiques. En règle générale, la personnalité de l'élève et son attitude vis-à-vis des valeurs fondamentales de notre société (démocratie, droits de l'homme, tolérance, solidarité, etc.) ne sont pas évaluées. Il en est de même pour les compétences et aptitudes (par exemple l'aptitude au travail en équipe, l'esprit autonome et critique, la maîtrise de certaines méthodes de travail systématique, l'aptitude à se débrouiller, à résoudre des problèmes) ainsi que la créativité et l'imagination. Bien sûr, ces critères sont très difficiles à évaluer. 
12 Le fait que certains pays, Länder, cantons et provinces laissent leurs écoles libres de concevoir les examens de fin d'études secondaires et d'en évaluer les résultats comme bon leur semble, tandis que d'autres possèdent un système de contrôle central où les épreuves sont fixées par le ministère, rend impossible toute comparaison des notes dans les examens finaux et porte atteinte à l'égalité des chances en Europe en ce qui concerne l'accès à l'enseignement supérieur.

13 Les systèmes d'évaluation actuels ont souvent tendance à négliger les aspects de motivation des élèves et leur auto-évaluation. Beaucoup d'élèves sont traumatisés par l'échec scolaire et perdent la joie d'aller à l'école et d'y apprendre, ce qui peut rendre l'enseignement inefficace et peu rentable.

14 Les universités ont tendance à se méfier de la valeur des notes scolaires et à introduire leurs propres examens d'entrée qui souvent font double emploi avec les examens de fin d'études secondaires - en dépit des résultats de recherches indiquant clairement que les notes scolaires ont, en règle générale, une grande valeur prédictive en ce qui concerne la réussite à l'université.

\section{L'atelier de Liège}

15 Le rapporteur général, le Professeur Georges Henry de l'université de Liège, avait tiré les conclusions suivantes des exposés et des discussions :

16 «Il importe d'encourager les études comparatives au niveau européen afin d'identifier les pratiques susceptibles de diminuer l'échec et de renforcer la motivation. Ces études permettraient de mettre en évidence le fait que certaines pratiques (par exemple, le redoublement), largement répandues dans certains pays, sont complètement inconnues dans d'autres.

17 «Si on veut diminuer les échecs et augmenter la qualité globale de l'enseignement, il faut se doter des moyens de piloter les systèmes scolaires. Ceci inclut notamment l'utilisation, comme en Suède, d'outils permettant la modération des notes.

18 "Le problème de la démotivation des élèves et des maîtres est crucial, car il atteint de très larges couches de la population scolaire et coûte fort cher sur le plan économique et social. Il est lié à la conception traditionnelle de l'école et de ses programmes, ainsi qu'à l'hétérogénéité croissante de la population scolaire. Des recherches, tant fondamentales qu'appliquées, devraient être entreprises dans les différents pays pour mieux cerner ces phénomènes

19 «Les programmes font une trop grande part à l'acquisition de connaissances parcellisées en un laps de temps donné. Des programmes moins ambitieux sur le plan des connaissances à acquérir, mais plus précis quant au niveau des objectifs cognitifs et affectifs à atteindre devraient être conçus et expérimentés. Il conviendrait d'être plus souple dans l'organisation temporelle des apprentissages et aussi de développer des procédures d'évaluation en rapport avec ces objectifs de haut niveau.

20 «Des procédures d'évaluation formative doivent être utilisées mais en évitant de se focaliser sur des objectifs trop étroits à atteindre en des temps trop courts.

21 "Il importe de donner plus de liberté, d'autonomie et de souplesse à l'institution scolaire, mais cela ne se traduira dans les faits qu'à la condition d'investir également dans la qualité des enseignants et dans le suivi des innovations. 
22 «Améliorer la qualité des enseignants suppose une meilleure formation initiale et un développement de leur motivation, ce qui ne sera possible que si l'on valorise leur rôle dans la société.

«La formation continue des enseignants doit également être renforcée dans certaines directions (participation, coopération, caractère pratique), favorables à l'acquisition de caractéristiques cognitives, affectives et sociales souhaitables. »

Outre les conclusions dégagées par le rapporteur général, le Secrétariat du Conseil de l'Europe avait attiré l'attention des ministres de l'Éducation sur les points suivants :

- la nécessité de l'évaluation des élèves comme outil de feedback pour les autorités de l'éducation sur l'efficacité du système scolaire ;

- la nécessité de l'évaluation comme un outil de feedback pour les élèves eux-mêmes, en vue de renforcer leur motivation. (La question est la suivante : de quel type de feedback a-t-on besoin pour que les élèves continuent d'être motivés? La recherche devrait permettre d'y répondre);

- l'effet des procédures traditionnelles d'évaluation rigoureuse qui ne peuvent aider que les bons élèves, mais qui démotivent les plus faibles. Dans ce cas, elle devient un obstacle à l'évolution souhaitée par les autorités et, notamment, leur désir de permettre à tous les élèves de développer au mieux leurs talents particuliers ;

- l'acquisition par les élèves d'aptitudes et de compétences qui ne sont pas évaluables ;

- l'introduction d'une expérience professionnelle à l'école pour aider à déterminer ce que les élèves sont capables de faire ;

- l'association étroite entre l'innovation dans les méthodes d'évaluation et l'élaboration du programme scolaire;

- la nécessité du dialogue régulier avec les parents comme élément important de l'évaluation et de la motivation;

- la nécessité d'aider les écoles à instaurer un climat propice qui incite les enseignants à s'engager dans de nouvelles méthodes d'évaluation et des façons inédites de répondre aux besoins des élèves ;

- la différenciation des objectifs qui peuvent, dans certains cas, différer selon les établissements et même selon les élèves, même si la nécessité d'un programme commun se fait sentir.

\section{L'atelier de Jyväskylä4}

Les conclusions suivantes méritent d'être soulignées. «Il faut faire une distinction entre les systèmes d'évaluation qui ouvrent l'accès à l'enseignement supérieur (le baccalauréat en France et l'Advanced level (GCE) en Angleterre et au pays de Galles) et ceux dont les résultats sont utilisés pour opérer une sélection. Pour évaluer le rôle d'examen de fin d'études, il faut savoir si c'est le premier, le deuxième ou les deux objectifs qui ont été choisis. L'influence de l'examen sur toute la scolarité dépend également de l'objectif de ce dernier. Il ne faut pas oublier que l'examen de fin d'études a d'autres finalités. En effet, de nombreux élèves ne suivent pas d'études supérieures et rentrent directement sur le marché de l'emploi et les employeurs cherchent donc dans les résultats à l'examen de fin d'études des données qui pourraient les aider à faire leur choix. Il peut y avoir un paradoxe, car ils recherchent d'une part des données qui leur permettent de mieux appréhender les qualités personnelles des élèves (capacité à travailler en équipe et à prendre des initiatives, stabilité, etc.) - ces capacités n'étant pas, le plus souvent, évaluées 
dans l'examen de fin d'études - et d'autre part, un moyen simple de choisir entre plusieurs candidats, une note unique par exemple. »

Les établissements scolaires ne pourront jamais renoncer à une certaine évaluation des élèves, mais il ne faudra pas oublier que le système d'évaluation a une influence inéluctable sur le processus d'enseignement et d'apprentissage. Il semble que trop souvent les écoles négligent de préparer leurs élèves à la vie dans une Europe intégrée en mettant plus au moins exclusivement l'accent sur des aspects étroits qui seront très probablement évalués dans les examens finaux. Des thèmes transversaux tels que l'éducation à la démocratie et au respect des droits de l'homme, l'éducation à la tolérance et la solidarité avec le Tiers Monde, à la responsabilité dans les relations sexuelles et à la responsabilité de parents, la sensibilisation aux problèmes de l'environnement, la compréhension des grands contextes historiques et culturels, la dimension européenne dans tout enseignement, la formation à l'esprit d'équipe, à la solution autonome et pluridisciplinaire de problèmes, à la communication, aux compétences sociales..., en souffrent.

27 Normalement, une bonne évaluation ne doit avoir qu'un seul objectif, cependant le plus souvent les systèmes d'évaluation sont conçus pour répondre à plusieurs besoins et des compromis doivent donc être faits.

Les notes scolaires devraient certifier si certains objectifs d'apprentissage sont atteints ; elles devraient aider les enseignants à arriver à un diagnostic du niveau de l'élève et donner aux élèves une idée des démarches à entreprendre pour se perfectionner. Actuellement, ceci n'est pas toujours le cas. «Souvent, l'accent est mis sur l'importance de l'objectif sommatif de l'évaluation - pour mesurer des acquisitions de l'élève à un moment donné - dans l'examen final. Cependant, l'évaluation formative remplit une fonction tout aussi importante, parce qu'elle fournit un bilan détaillé qui permet à l'enseignant de déterminer les prochaines étapes que l'élève devra suivre. Cette fonction est généralement placée sous la responsabilité de l'enseignant et porte les appellations d'évaluation interne, d'évaluation de l'enseignant ou d'évaluation diagnostique. Les enseignants ont besoin de beaucoup de soutien au sein de ce processus, aussi bien en termes d'outils et de manuels que de formation. Les avis diffèrent quant aux outils : pour certains, il faut aider les enseignants à améliorer leurs tests, pour d'autres, il faut les former à des méthodes d'évaluation informelles : entretiens, projets spéciaux, utilisation des travaux faits à la maison et en classe. Il semble que seuls l'Angleterre et le pays de Galles aient tenté d'intégrer évaluation sommative et formative en un seul système, l'expérience s'avérant difficile à mener. »

Un nouvel objectif de l'évaluation est de mesurer le rendement des établissements scolaires dans leur ensemble :

Certains pays sont en train de mettre ou ont déjà mis au point des programmes nationaux d'évaluation dont les objectifs sont plus vastes. En France, les élèves sont soumis à une évaluation de français et de mathématiques au cours de leurs sixième et onzième années de scolarité. Ces évaluations fournissent un diagnostic aux enseignants en leur permettant de mieux comprendre les normes nationales et constituent, un moyen de contrôle interne de l'efficacité des écoles. En Angleterre et au pays de Galles, on propose que les résultats de l'évaluation nationale soient transmis aux parents (en ce qui concerne leur enfant) et publiés sous forme comparative pour l'ensemble de l'école. Tous les résultats sont analysés au niveau national. 
Il y a une tendance en Europe à passer de l'évaluation à référence normative à l'évaluation à référence critérielle et de combiner des éléments d'évaluation interne de l'école avec des éléments d'une évaluation externe. Il faudra que l'évaluation à référence critérielle " permette aux enseignants et aux autres de déterminer avec précision ce qui doit être enseigné et quelles connaissances ou compétences doivent avoir les élèves pour un résultat donné. La principale difficulté soulevée par ce type d'évaluation réside dans la formulation de critères suffisamment précis pour permettre une évaluation et suffisamment généraux pour qu'ils ne soient pas trop nombreux. "

31 Les enseignants doivent être bien renseignés sur les objectifs du système d'évaluation et des procédures d'examen en vigueur dans leur pays. Il faudrait les associer à l'élaboration du système et de ses réformes.

32 L'examen de fin d'études secondaires devrait certifier une bonne culture générale ainsi qu'une vaste gamme d'aptitudes et de compétences, nécessaires à la fois pour la poursuite des études au niveau supérieur et à la vie d'adulte en général (concept de la maturité). Dans la pratique de beaucoup de pays, ceci est devenu une fiction à cause du phénomène de l'enseignement de masse (aux niveaux de l'enseignement secondaire, deuxième degré, et de l'enseignement supérieur) et de la liberté que les élèves ont de ne se concentrer que sur deux ou trois matières-clé pendant leurs dernières années à l'école. Même si la convention du Conseil de l'Europe de 1953 le stipule, les certificats de fin d'études secondaires ne sont plus toujours équivalents à travers toute l'Europe.

En conséquence, le nombre des universités ayant fixé leurs propres exigences d'accès et organisant leurs propres examens ou concours d'entrée a augmenté. Il n'y a rien de mauvais dans ce développement pourvu que l'évaluation scolaire et la sélection à l'entrée aux universités soient clairement séparées et que ceci ne donne pas aux établissements scolaires un prétexte pour ne faire rien d'autre que de préparer leurs élèves à ces examens d'entrée à l'université. Parfois, les universités semblent inquiètes de la baisse du niveau scolaire et essayent donc d'imposer des normes plus élevées par le biais de leurs procédures d'admission. Les résultats de recherche, toutefois, indiquent clairement que les notes scolaires constituent une base fiable pour la prédiction de la réussite universitaire (mais pas professionnelle!). Le type de culture générale et d'aptitudes et compétences à tester à l'école est aussi pertinent pour l'enseignement supérieur que pour la vie active et le marché de travail.

Des efforts pour arriver à des critères et des échelles comparables en Europe sont à encourager. Pour l'enseignement et l'apprentissage des langues vivantes, par exemple, le Conseil de l'Europe est en train de développer de tels critères et échelles. Il faudra, toutefois, ne pas perdre de vue le fait que la valeur de l'évaluation scolaire restera toujours relative et souvent elle sera assez subjective. Il ne faudra pas la surestimer. En tous cas, il serait utile de remplacer des notes sommaires par matière ou discipline par une évaluation plus détaillée et plus fine de différents éléments d'un programme (tels que prononciation, expression orale et écrite, qualité des traductions vers la langue maternelle, vocabulaire, grammaire, compréhension de la culture des pays étrangers, en ce qui concerne les langues étrangères). L'essentiel est de bien préparer les jeunes à l'Europe de demain, pour qu'ils deviennent des citoyens responsables, tolérants, ouverts au monde, polyglottes, débrouillards, pleins d'imagination et de créativité. Le meilleur élève de la dernière promotion de quelque établissement d'élite ne fera pas son chemin en Europe, s'il reste un égoïste qui a du mal à s'intégrer dans une équipe internationale et 
qui ne voit les choses que du point de vue parfois étroit de son propre pays. "Non scolae sed vitae discimus » ( «nous n'apprenons pas pour l'école mais pour la vie ») et, en dernière instance, ce sera la vie et non pas l'école qui donnera des notes.

\section{NOTES}

1. Les 1-15 septembre 1989 sur l'évaluation des résultats scolaires : motivation et réussite des élèves.

2. Les 15-18 juin 1993 sur l'évaluation des élèves et le rôle de l'examen final.

3. La version anglaise des actes des deux ateliers a été ensuite publiée sous forme de livre, par la Maison Swets \& Zeitlinger à Lisse en Hollande. La version française des rapports peut être demandée à la section de la Recherche, Conseil de l'Europe, F-67075 Strasbourg Cedex.

4. Document du Conseil de l'Europe DECS/Rech (93) 50, se basant sur le résumé du rapporteur général M. Chris Whetton (Angleterre).

\section{RÉSUMÉS}

Dans de nombreux pays européens, des recherches en éducation ont eu pour but de comparer les différents systèmes d'évaluation et d'en examiner la validité. Le Conseil de l'Europe a pris l'initiative de tirer un bilan de ces travaux. Cet article présente les principales indications et recommandations fournies aux décideurs en éducation à l'issue de ce bilan.

\section{INDEX}

\section{Index géographique : Europe}

Mots-clés : analyse comparative, Conseil de l'Europe, évaluation, recherche comparative, évaluation de l'élève

\section{AUTEUR}

\section{MICHAEL VORBECK}

Chef de la section de la recherche pédagogique, Conseil de l'Europe, direction de l'Éducation, de la Culture et du Sport, Strasbourg, France 